\title{
PERSONAS CON DIABETES: ADHERENCIA AL TRATAMIENTO, CALIDAD DE VIDA Y EDUCACIÓN
}

\author{
PEOPLE WITH DIABETES: ADHERENCE TO TREATMENT, \\ QUALITY OF LIFE AND EDUCATION
}

La evidencia disponible en la literatura muestra que la calidad de atención de las personas con diabetes, tanto a nivel nacional como internacional, dista mucho de ser la ideal ${ }^{1-3}$, lo que determina el desarrollo y progresión de las complicaciones crónicas prevenibles de la enfermedad. Esto redunda en un aumento de los costos de su atención y disminución de la calidad de vida de quienes la padecen ${ }^{4}$.

Lograr un cambio efectivo de esta situación implica, entre otros objetivos, identificar las causas subyacentes que la determinan. En este sentido en la diabetes y los factores de riesgo asociados, al igual que toda enfermedad crónica, la calidad de atención depende en gran parte de la interacción efectiva entre el médico y el paciente, es decir de un correcto diagnóstico y prescripción apropiada, además de una correcta implementación de la misma. A esta última la solemos denominar adherencia del paciente y la han destacado diversos autores a nivel internacional ${ }^{5}$. Por el contrario no existen demasiadas evidencias nacionales al respecto y cabe destacar que un grupo de diabetólogos argentinos, trabajando en forma cooperativa, en esta edición presenta evidencias locales registradas en diferentes zonas del país sobre el tema (artículos de la Dra. Linari y col.).

El grupo mencionado, con el empleo de cuestionarios estandarizados y validados, demuestra que dicha adherencia, si bien no es significativamente diferente de la descripta en la literatura (aproximadamente el 60\%), explica al menos en parte la deficiente calidad de atención verificada a nivel nacional e internacional.
En los estudios mencionados se destaca que el déficit de adherencia a las prescripciones es heterogéneo y no necesariamente asociado a su costo o dedicación para implementarla. Así por ejemplo se verificó mayor adherencia al automonitoreo glucémico (AMG) en pacientes tratados con insulina y menor en aquellos tratados con dieta, ejercicio y uso de antidiabéticos orales, e igualmente mayor adherencia en el cuidado de los pies aún comparada con el registrado a nivel internacional.

Dado que aparentemente en este estudio el costo y la dedicación -en el caso del AMG- no serían los principales motores de la diferente adherencia, cabe entonces preguntarse por otros posibles condicionantes de esta heterogeneidad en la adherencia a la prescripción. Una alternativa posible sería el tiempo dedicado por el médico y su equipo a explicar su realización y cómo utilizar los resultados del AMG para adaptar la dosis de insulina o las formas de realizar los cuidados del pie. En ambos casos presumiblemente la educación del paciente desempeñaría un rol fundamental ${ }^{6}$. No menos importante sería el valor otorgado por el paciente a dicha práctica: comprobación directa del mejor control metabólico en el primer caso y menor desarrollo de lesiones respecto del cuidado de los pies?.

Aunque al igual que en las conclusiones de los trabajos, los autores reclaman desarrollar nuevos estudios para aseverar la fortaleza de las mismas, y que las interpretaciones merecen demostrarse mediante nuevas investigaciones.

Aún con debilidades y falencias propias de todo estudio clínico, los resultados aportados en esta edi- 
ción deberían servir como un primer diagnóstico de situación basado en la evidencia, para luego definir e implementar estrategias que apunten a corregir las falencias descriptas. Dado que lo planteado implica un gran esfuerzo, quizás debamos recurrir como estímulo a una vieja frase de Elliot P. Joslin que hace varios años sabiamente planteó: "El diabético que más sabe, vive más y mejor tiempo"8.

Juan José Gagliardino Investigador Superior del CONICET, Centro de Endocrinología Experimental y Aplicada (CENEXA), Facultad de Ciencias Médicas, Universidad Nacional de La Plata

\section{BIBLIOGRAFÍA}

1. Reach G, Pechtner V, Gentilella R, Corcos A, Ceriello A. Clinical inertia and its impact on treatment intensification in people with type 2 diabetes mellitus. Diabetes Metab 2017; 43:501

2. Tabesh M, Magliano DJ, Tanamas SK, Surmont F, Bahendeka S, Chiang CE, Elgart JF, Gagliardino JJ, Kalra S, Krishnamoorthy S, Luk A, Maegawa H, Motala AA, Pirie F, Ramachandran A, Tayeb K, Vikulova O, Wong J, Shaw JE. Diabetes management and treatment approaches outside of North America and West Europe in 2006 and 2015. Acta Diabetol 2019; 56:889

3. Gagliardino JJ, Elgart JF, Forti L, Querzoli I, Chantelot JC. Type 2 diabetes: Prescription patterns and treatment outcomes of IDMPS survey in Argentina. Diabetes Res Clin Pract 2019; 153:86

4. Elgart JF, Asteazarán S, De La Fuente JL, Camillucci C, Brown JB, Gagliardino JJ. Direct and indirect costs associated to type 2 diabetes and its complications measured in a social security institution of Argentina. Int $\mathrm{J}$ Public Health 2014; 59:851
5. Mogre V, Johnson NA, Tzelepis F, Shaw J, Paul C. Adherence to self-care behaviours and associated barriers in type 2 diabetes patients of low-and middleincome countries: a systematic review protocol. Syst Rev 2017; 6:39.

6. Assal JP. Revisiting the approach to treatment of longterm illness: from the acute to the chronic state. A need for educational and managerial skills for long-term foIlow-up. Patient Educ Couns 1999; 37:99 (Review).

7. Loskutova NY, Tsai AG, Callen E, Ajayi K, Carroll JK, Harrington $\mathrm{M}$, TurnerTJ, Pace WD. Differences in perspectives regarding diabetes management between health care providers and patients. Transl Behav Med 2018; 8:328

8. Barnett D, Joslin EP. A centennial portrait. Joslin Diabetes Center 1999. 